\title{
Detraction the Clutter for the Best Binary Phase Codes that Begot By Genetic Algorithm Using Wiener Filter
}

\author{
W. T. Ali
}

\begin{abstract}
This work illustrates the performance of detraction of clutter (unwanted echo signals, which mask the desired target signal and make difficult to detect the target) as a result of binary codes generating by genetic algorithm with length up to 105 bits, with use the minimum peak sidelobes as criteria for generation codes. Then, for further reduction of sidelobes, the mismatched optimum integrated sidelobe level filter (Wiener filter) is used with and without White Gaussian Noise. When the mismatched filter is used without noise, then the reduction of sidelobe level has the improvement of peak sidelobe level value on the average (4-15) $\mathrm{dB}$, and for integrated sidelobe level value on the average (5-17) $\mathrm{dB}$, which depends on the code length while it is accompanied by, signal to noise ratio loss level in range $(0.2-1.4) \mathrm{dB}$. On other hand, when the mismatched filter is used with noise, the signal to noise ratio loss level is in range $(0.2-1.4) \mathrm{dB}$.
\end{abstract}

Index Terms-Minimum Peak Sidelobe (MPS), wiener filter (ISL) filter, Autocorrelation Function (ACF), Genetic algorithm (GA.), Signal to Noise Ratio Loss (SNRL)..

\section{INTRODUCTION}

The use of high energy (long pulses duration) permit high detection range and the time-compressing of a received radar signal permitted an increase in the range resolution capability of radar. In that way the radar will utilize a long pulse to achieve large radiated energy, but simultaneously it will be obtain the range resolution of a short pulse. Pulse compression can be accomplished by employing frequency or phase modulation to widen the signal bandwidth.

Then using a matched filter (also called a pulse compression filter) or any other mismatched filter on reception to compress the pulse [1].

Phase coding can be divided into two types: binary phase coding and polyphase coding. The term "range sidelobes" was used first to describe the lobes in both sides of a mainlobe of the compressed time function of a received binary phase-coded signal. The term sidelobe is generalized to describe the time function of any pulse compression waveform.

Optimal binary codes are binary sequences whose peak sidelobes of the aperiodic autocorrelation function is the minimum possible for a given code length. Codes whose autocorrelation function or zero-Doppler response exhibit low sidelobes are desirable for pulse compression radars because the clutter rejection is satisfied when the pulse

Manuscript published July 31, 2016.

Weaam Talaat Ali is with the College of Engineering, Diyala University, Diyala, Iraq (wface2010@yahoo.com). compression waveforms have low sidelobes [2].

By exhaustive computer search program, Lindner [3] found all binary sequences up to length 40 with minimum PSL. Cohen et al. [4] extended those results to length 48 . Boehmer [5] has developed an analytical technique for generating good binary pulse compression codes.

In this paper, the sequences generated by genetic algorithm up to length 105 is optimum, to suppress these sidelobes, the amplitude response of the matched filter is reshaped by another filter (optimum mismatched ISL filter), wiener filter, to yield lower sidelobes (to reduce the clutter as a result) at the expense of some signal-to-noise ratio loss at the output of the mismatched filters.

\section{GENETIC Algorithm}

\section{A. Optimization with Genetic Algorithm}

A genetic or evolutionary algorithm employs the principles of evolution found in nature to the problem of finding an optimal solution to a problem solver. In a "genetic algorithm" the problem is encoded in a series of bit strings that are manipulating by the algorithm, in it the decision variables and problem functions are used immmeditly.it is different from "classical" optimization methods in many ways [6]:

1) Random versus deterministic operation.

2) Population versus single best solution.

3) Creating new solution through mutation.

4) Combining solution through crossover.

5) Selecting solution via survival of the fittest.

6) Drawbacks of evolutionary algorithms.

\section{B. Algorithm Generating Binary Phase Codes by Genetic Algorithm}

The output of the matched filter is the ACF of the input signal (without any Doppler shift frequencies and considering noise). So, a good criterion on the optimum binary phase code is that its autocorrelation has sidelobes as minimum as possible. Therefore, it is desired to find N-bit binary sequences whose Minimum Peak Sidelobe (MPS) has the minimum value among all $2^{\wedge} \mathrm{N}$ existing codes.

Fig. 1 shows a flow chart of generation the binary sequences by genetic algorithm and the execution of GA discussed by insertion initial population randomly included casual $\mathrm{N}$-element codes which represent the initial binary phase vectors, then, calculate the MPS as a fitness function for each sequence (chromosome) and then if any of calculated sequences (chromosomes) are greater than desired MPS, then these sequences are omitted from the search space.

Omit all Allomorphic forms of each sequence to reduce the search space. 
Then, enter the sequences in step of selection by remain the best code and eliminate the worse code according to the roulette wheel selection that is used in this work.

Later, the sequences must pass through crossover operating as a single point crossover, with a specified the crossover probability $\mathrm{P}_{-} \mathrm{c}$ equal to 0.1 then to a single point mutation with a specified the mutation probability $\mathrm{P}_{-} \mathrm{m}$ equal to 0.1 , which are used in this work.

After that, the individual if it found must replaced by the offspring sequences to calculate MPS again to the decision procedure of (selection, crossover and mutation) retry or ended according to the stopping criteria.

If the decision goes to yes, the procedure is ended and the sequence produced is according to MPS required; otherwise, the sequences return to the process (selection, crossover and mutation) to result the best code that wanted.

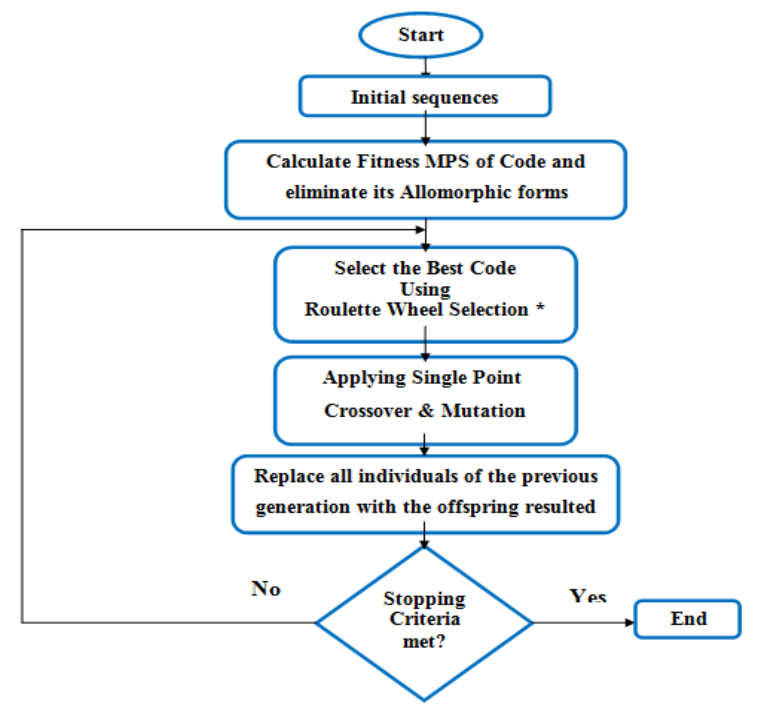

Fig. 1. A flow chart of Genetic algorithm to generate Binary Phase Codes.

\section{WIENER FILTER}

An optimal integrated sidelobe level (ISL) filter, or Wiener filter, is a filter used to minimize the total energy in the range sidelobes [9].

Optimal ISL filter is such a Finite-Duration Impulse Response (FIR) filter that it can be expressed as a matrix operation:

Let $c=[c(0), c(1), c(2), \ldots, c(N-1)]$

and,

$$
\mathrm{h}=[\mathrm{h}(0), \mathrm{h}(1), \mathrm{h}(2), \ldots ., \mathrm{h}(\mathrm{P}-1)]
$$

where the row vector $\mathrm{c}$ represents the known code of length $\mathrm{N}$, and the row vector $\mathrm{h}$ contains the unknown filter coefficients of length $\mathrm{P}$, respectively.

\footnotetext{
* The most common method of selection is the Roulette Wheel Selection. By this method, parents are selected according to their fitness. The better chromosomes are, the more chances to be selected, a roulette wheel can be imagined, where all the chromosomes in the population are placed. The size of the section in the roulette wheel is proportional to the value of the fitness function of every chromosome. The section represents a particular chromosome, which gets larger as the corresponding fitness value gets bigger than other chromosomes' fitnesses [8].
}

The filter coefficients are real and the filter length $\mathrm{P}$ can be longer than the code length $\mathrm{N}$, i.e., $\mathbf{P} \geq \mathbf{N}$. When this is the case, the code vector is zero-padded to match the length of the filter. For the sake of simplification, if $\mathrm{N}$ is even then $\mathrm{P}$ is even too, and when $\mathrm{N}$ is odd then $\mathrm{P}$ is odd too. Thus, $\mathrm{P}$ $\mathrm{N}$ is always even, since $(\mathrm{P}-\mathrm{N}) / 2$ is an integer denoted as $\mathrm{Z}$. Now, $\mathrm{Z}$ is defined as an all-zero code of length $\mathrm{Z}$ used to create a zero-padded signal code of length $\mathrm{P}=\mathrm{N}+2 \mathrm{z}$ given by matrix [10]:

$$
\mathrm{X}=\left[\begin{array}{lll}
Z & \mathrm{c} & \mathrm{Z}
\end{array}\right]
$$

The aperiodic crosscorrelation output at tap m, due to the zero-padded input code, is given by [10]:

$$
\mathrm{y}(\mathrm{m})=\sum_{\mathrm{r}=0}^{\mathrm{P}-1} \mathrm{x}(\mathrm{r}) \mathrm{h}(\mathrm{r}-\mathrm{m}) \quad \mathrm{m}=-(\mathrm{P}-1), . .,(\mathrm{P}-1)
$$

where vectors, $\mathrm{x}$ and $\mathrm{h}$, are defined to be zero for taps less than zero or greater than (P-1). Equation (2) can be represented in matrix form as [10]:

$$
\mathrm{y}=\mathrm{hX}
$$

where $\mathrm{X}$ is the $(\mathrm{P})$ by $(2 \mathrm{P}-1)$ Hankel matrix of the zeropadded code vector $\mathrm{x}$, and $\mathrm{y}$ is the crosscorrelation vector of length (2P-1). The Hankel matrix X is defined as [11]:

$$
\mathrm{X}=\left[\begin{array}{cccccc}
0 & \cdots & 0 & \mathrm{x}(0) & \mathrm{x}(1) & \mathrm{x}(\mathrm{P}-1) \\
\vdots & 0 & \mathrm{x}(0) & \mathrm{x}(1) & \cdots & 0 \\
0 & \mathrm{x}(0) & \mathrm{x}(1) & \cdots & \mathrm{x}(\mathrm{P}-1) & \vdots \\
\mathrm{x}(0) & \mathrm{x}(1) & \cdots & \mathrm{x}(\mathrm{P}-1) & 0 & 0
\end{array}\right]
$$

and the crosscorrelation vector $\mathrm{y}$ is defined as :

$$
\mathrm{y}=[\mathrm{y}(-(\mathrm{P}-1)) \ldots \ldots \ldots . . . \mathrm{y}(0) \ldots \ldots \ldots . \mathrm{y}(\mathrm{P}-1)]
$$

In calculating the total power in the sidelobes, the main peak $y(0)$ term must be subtracted out. Therefore, the total power in the sidelobes is given by:

$$
E=\sum_{m=-(P-1)}^{(P-1)} y^{2}(m) f^{2}(m)-y^{2}(0) f^{2}(0)
$$

In minimizing $\mathrm{E}$, the trivial solution, $\mathrm{h}(\mathrm{r})=0$ where $\mathrm{r} \in$ $[0, \ldots,(\mathrm{P}-1)]$ must be disallowed, so a constraining equation, defining the peak as constant, is used, as follows:

$$
\sum_{r=0}^{\mathrm{P}-1} \mathrm{x}(\mathrm{r}) \mathrm{h}(\mathrm{r})-\mathrm{N}=0
$$

\section{The Performance of Sidelobe Reduction TECHNIQUES}

The following measures are often used to quantify the 
performance of range sidelobe suppression techniques. These measures are as in the following [12]:

\section{A. Peak Sidelobe Level:}

Peak Sidelobe Level, a measure of the largest sidelobe power as compared with the mainlobe power, is defined as [10]:

$$
\text { PSL }=10 \log _{10}\left[\frac{\text { peak sidelobe power }}{\text { total mainlobe power }}\right]
$$

\section{B. Integrated Sidelobe Level:}

Integrated Sidelobe Level, a measure of the energy distributed in the sidelobes as compared with the mainlobe power, is defined as [10]:

$$
\text { ISL }=10 \log _{10}\left[\frac{\text { power integrated over sidelobes }}{\text { total mainlobe power }}\right]
$$

\section{Mismatch Loss:}

SNRL is expressed in decibels as the ratio of the peak output value of the mismatched filter $y_{\text {peak,mismatched }}$, relative to the peak output value of the matched filter $\mathrm{y}_{\text {peak'matched }}$, as follows [10]:

$$
\text { SNRL }=-20 \log _{10}\left[\frac{\mathrm{y}_{\text {peak,mismatched }}}{\mathrm{y}_{\text {peak,matched }}}\right]
$$

where the coefficients of both filters have been normalized for unity noise gain, such that:

$$
\sqrt{\sum_{\mathrm{r}=0}^{\mathrm{P}-1}[\mathrm{~h}(\mathrm{r})]^{2}}=1
$$

Or,

$$
\mathrm{SNRL}=-20 \log _{10}\left[\frac{\mathrm{y}_{\text {peak,mismatched }}}{\mathrm{N}}\right]
$$

where $\mathrm{N}$ is the code length, and the coefficients of the mismatched filter are normalized to yield the same noise output power as the matched filter, when the input is only white noise. Thus, the normalization requires that [12]:

$$
h h^{\mathrm{T}}=\mathrm{xx}^{\mathrm{T}}
$$

where $\mathrm{h}^{\mathrm{T}}$ is the transpose of $\mathrm{h}$ vector.

\section{Simulation And Results}

\section{A. Best Binary Codes Generating by GA.}

The criterion for chosen optimum codes is MPS for each sequence for a certain length and according to the flow chart in Fig.1. From simulation an 105 codes were obtained and in

\begin{tabular}{|c|c|c|c|c|}
\hline $\begin{array}{l}\text { Code } \\
\text { Length } \\
(\mathrm{N})\end{array}$ & MPS & Sequence & PSL $[\mathrm{dB}]$ & ISL $[\mathrm{dB}]$ \\
\hline 3 & 1 & 110 & -9.542 & -6.532 \\
\hline 8 & 2 & 10010111 & -12.041 & -6.020 \\
\hline 9 & 2 & 011010111 & -13.064 & -5.282 \\
\hline 13 & 1 & 1111100110101 & -22.278 & -11.487 \\
\hline 14 & 2 & 01010010000011 & -16.901 & -7.124 \\
\hline 15 & 2 & 001100000101011 & -17.501 & -6.894 \\
\hline 24 & 3 & 011001001010111111100011 & -18.061 & -9.030 \\
\hline 25 & 2 & 1001001010100000011100111 & -21.938 & -8.514 \\
\hline 36 & 3 & $\begin{array}{c}001100110001010010100000100 \\
000111110\end{array}$ & -21.583 & -8.384 \\
\hline 41 & 3 & $\begin{array}{c}000111000111010100101001000 \\
00001101100100\end{array}$ & -22.713 & -8.753 \\
\hline 42 & 3 & $\begin{array}{c}000100010001000111101110000 \\
111010010110100\end{array}$ & -22.922 & -9.411 \\
\hline 49 & 4 & $\begin{array}{c}000010010101010111110110001 \\
1110011110010001101111\end{array}$ & -21.762 & -5.478 \\
\hline 57 & 4 & $\begin{array}{c}000010010011010001010100011 \\
101101011000100011110001101 \\
111\end{array}$ & -23.076 & -6.4962 \\
\hline 64 & 4 & $\begin{array}{c}010000001001000010100010111 \\
010011110011000110010001101 \\
1111000010\end{array}$ & -24.082 & -7.225 \\
\hline 77 & 4 & $\begin{array}{c}110010110000110101110001001 \\
010001110111001001010100001 \\
11111011101101111110100\end{array}$ & -25.688 & -7.4117 \\
\hline 80 & 4 & $\begin{array}{c}110011100100001111001000110 \\
1100110000110010011101101 \\
101011011111110010010 \\
0101111\end{array}$ & -26.020 & -7.9588 \\
\hline 90 & 5 & $\begin{array}{c}110011001001101101100001111 \\
1000011101010010001 \\
00010110110101 \\
101111101010000100001000010 \\
001\end{array}$ & -25.10 & -7.568 \\
\hline 100 & 5 & $\begin{array}{c}11011111010010010000 \\
1010111111111 \\
00000111111000001110010 \\
0010011111110010100101010 \\
0110101011011001101\end{array}$ & -26.020 & -7.5746 \\
\hline
\end{tabular}
Table I a samples of these codes are presented. In this Table, the codes are stated after excluding all Allomorphic forms of codes during generation, and PSL, ISL exhibited in this Table according to (8) and (9) respectively for each code.

TABLE I: SAMPLES OF OPTIMUM BinARY CODES RESUlted By GA.

\section{B. Wiener Filter for Binary Phase Codes with and without} Noise:

In this section, examples on the free noise effect are introduced. In order to test the chosen codes under the same conditions, the filter length $(\mathrm{P})$ is considered to be (three times of the code length)-elements in all sections below.

In Table II some codes chosen from Table I as shown 
entered as input to the optimum ISL filter and the results of PSL, ISL, SNRL is presented in this Table with noise free,

TABLE II: OUTPUT OF OPTIMUM MisMATCH (ISL) FILTER FOR SOME

\begin{tabular}{|c|c|c|c|}
\hline $\begin{array}{l}\text { Code } \\
\text { Length } \\
(\mathrm{N})\end{array}$ & PSL $[\mathrm{dB}]$ & ISL [dB] & SNRL [dB] \\
\hline 8 & -22.400 & -11.371 & 0.955 \\
\hline 13 & -38.470 & -30.033 & 0.204 \\
\hline 14 & -22.818 & -14.381 & 1.130 \\
\hline 25 & -34.023 & -24.730 & 0.650 \\
\hline 36 & -26.828 & -17.335 & 0.714 \\
\hline 49 & -25.354 & -13.008 & 1.375 \\
\hline 57 & -28.960 & -15.076 & 1.048 \\
\hline 80 & -33.824 & -19.066 & 0.802 \\
\hline 90 & -33.352 & -18.642 & 0.9679 \\
\hline 100 & -33.916 & -18.585 & 0.973 \\
\hline
\end{tabular}

By comparing the result of Table II with the result of Table I, an improvement in values of PSL, ISL is found with disadvantage of SNRL due to mismatch optimum (ISL) filter when the noise is free, The reduction of sidelobe level has the improvement of PSL value on the average (4-15) dB, and for ISL value on the average (5-17) $\mathrm{dB}$, Which are depend on the code length while it is accompanied by, SNRL in range (0.2-1.4) $\mathrm{dB}$.

Fig.2 shows the normalized crosscorrelation of the optimum mismatch ISL filter when it is applied to $\mathrm{N}=57$ bits binary code (as example) in noise free, PSL improves from $-23.0763 \mathrm{~dB}$ to $-28.9602 \mathrm{~dB}$, ISL improves from $6.4962 \mathrm{~dB}$ to $-15.0764 \mathrm{~dB}$, and SNRL equal to $1.0487 \mathrm{~dB}$.

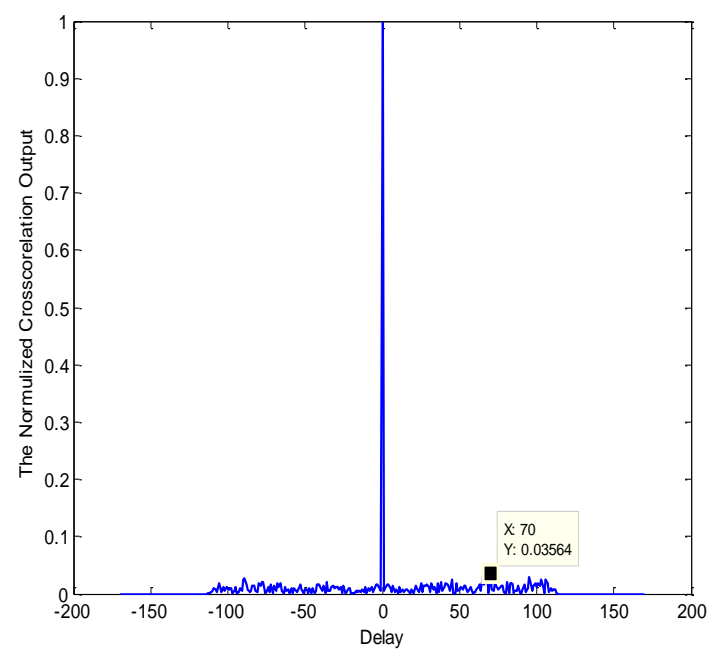

Fig. 2.The normalized Crosscorrelation of the optimal ISL filter when it is applied to $\mathrm{N}=57$-bit binary code in noise free.
Fig. 3 shows the PSL-versus-P (P-filter length) behaviors of the optimal ISL filter when it is applied to five different binary phase codes with SNRi equal to $7 \mathrm{~dB}$. While, Fig. 4 show the ISL-versus-P behaviors of the optimal ISL filter when it is applied to five different binary phase codes with SNRi equal to $7 \mathrm{~dB}$. Moreover, Fig.5 shows the SNRLversus-P behaviors of the optimal ISL filter when it is applied to five different binary phase codes with SNRi equal to $7 \mathrm{~dB}$.

Fig. 6 shows PSL-versus-SNRi behavior, Fig. 7 shows ISL-versus-SNRi behavior and Fig. 8 shows SNRL-versusSNRi behavior of the optimal ISL filter when it is applied to five different binary phase codes with different SNRi.

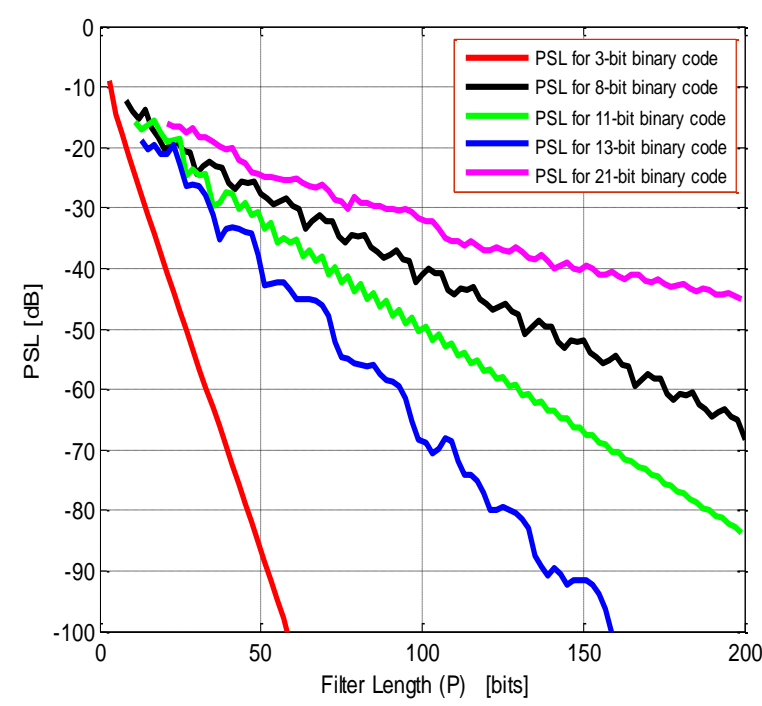

Fig. 3. PSL-versus-P behavior of the optimal ISL filter when it is applied to five different codes with a SNRi $=7 \mathrm{~dB}$.

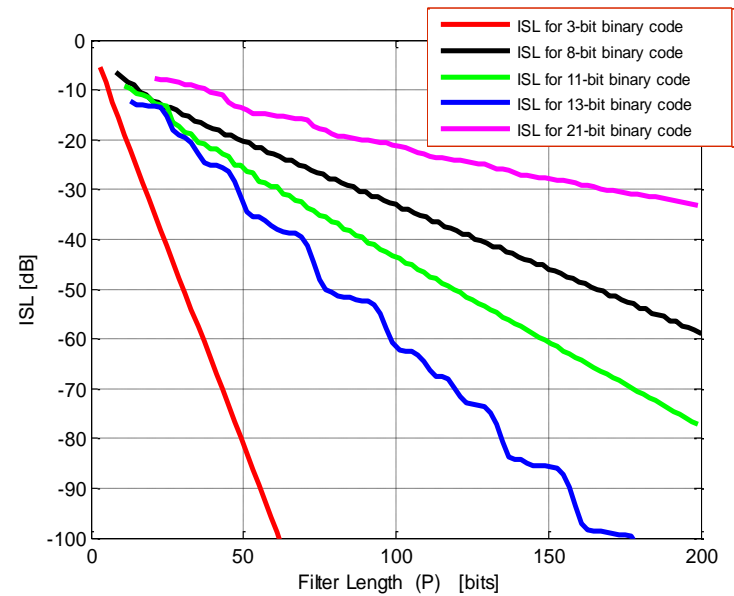

Fig. 4. ISL-versus-P behavior of the optimal ISL filter when it is applied to five different codes with a SNRi $=7 \mathrm{~dB}$. 


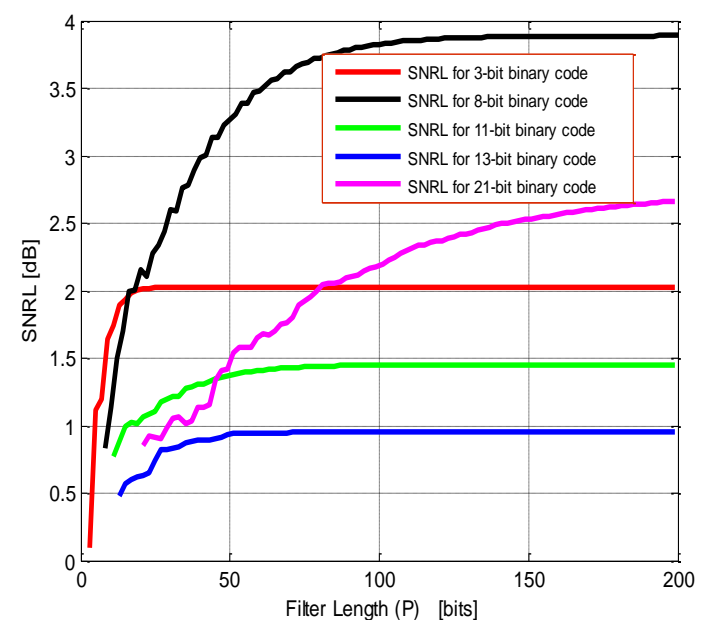

Fig. 5. SNRL-versus-P behavior of the optimal ISL filter when it is applied to five different codes with a $\mathrm{SNRi}=7 \mathrm{~dB}$.

From the previous Fig. 3, 4 and 5, it is believed that PSL, ISL improve when the filter length is increase, but SNRL increases as disadvantage.

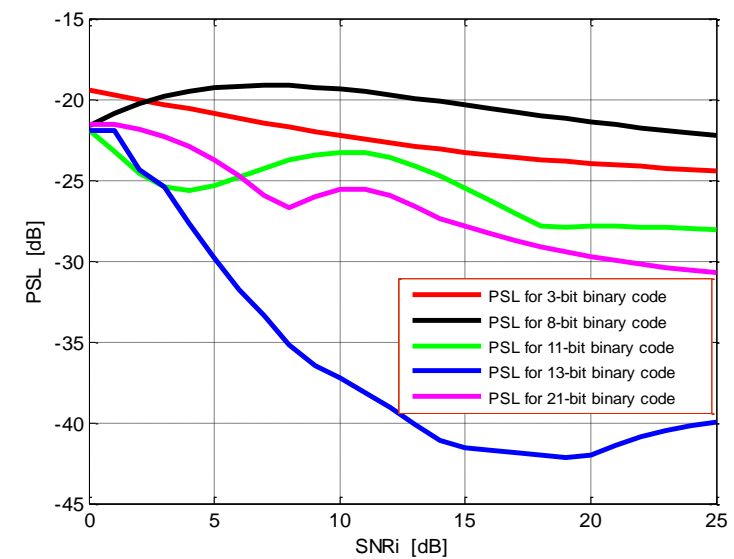

Fig. 6. PSL-versus-SNRi behavior of the optimal ISL filter when it is applied to five different codes with different SNRi.

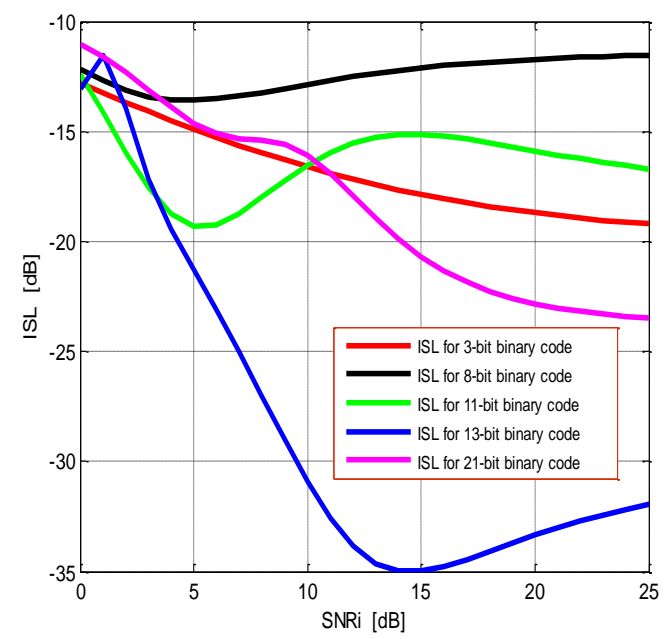

Fig. 7. ISL-versus-SNRi behavior of the optimal ISL filter when it is applied to five different codes with different SNRi.

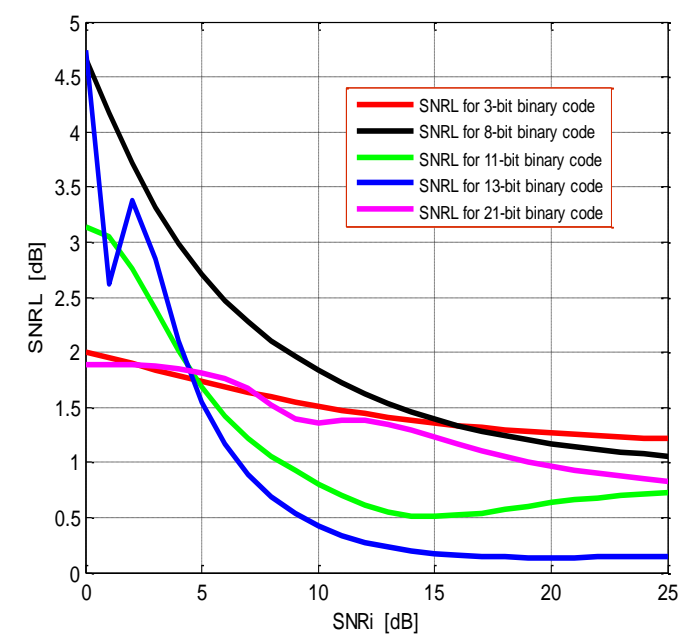

Fig. 8: SNRL-versus-SNRi behavior of the optimal ISL filter when it is applied to five different codes with different SNRi.

From the previous Fig. 6, 7 and 8, it is believed that when the SNRi increases the PSL, ISL has a small improvement at the same time the SNRL decreases very large (the range of SNRL is $(0.2-1.4) \mathrm{dB}$ ), which are depend on the code length. So, the best result of SNRL is up to $5 \mathrm{~dB}$ of SNRi. The range of SNRL is $(0.2-1.4) \mathrm{dB}$.

\section{CONCLUSION}

The followings summarize the main important points that are noted from this work.

1) For the generated optimum codes by the programmed GA., the range of code length from (2-105) bits, the limitation of the upper limit is related to the time consuming.

2) The codes which are generated by the programmed GA show that, MPS increases with the increase in code length. As a result, the value of PSL is also increased.

3) The quality of solution improves with increase in the number of generations.

4) When the filter length is increase, The PSL, ISL improves very well, but SNRL increases as disadvantage.

5) When the SNRi increases the PSL, ISL has a small improvement at the same time the SNRL decreases very large as advantage.

\section{REFERENCES}

[1] O. Aktop, “ Optimization of Doppler Processing by Using Bank of Matched Filters", M.S. Thesis, Middle East Technical Univ. ,Turkish, The Graduate School Of Natural And Applied Sciences, September 2005.

[2] M. Skolnik, Radar Handbook, Mc Graw-Hill, Tokyo, 3rd ed. 2008,pp.581

[3] J. Lindner, "Binary sequences up to length 40 with best possible autocorrelation function", Electron. Lett. vol. 11, no. 21, pp. 507, October 1975

[4] M. N. Cohen, G. E. Coxson, and A. Hirschel, "New Results on Minimum-PSL Binary Codes", IEEE National Radar Conference, Atlanta, GA, pp. 153-156, May 2009.

[5] S. Ananji, "New approaches of Pulse Compression Techniques of phase-coded Waveforms in Radar", M.S. Thesis, Electronics \& Communication Eng. Dept. College of Eng. National Institute of Technology, India, 2010. 
[6] Q. Wang, P. Spronck, and R. Tracht, "An Overview of Genetic Algorithms Applied to Control Engineering Problems", IEEE, Proc. of the Second International Conference on Machine Learning and Cybernetics, china, 2-5 November 2003.

[7] M. Skolnik, Radar Handbook, Mc Graw-Hill, Tokyo, 2nd ed, 1990.

[8] D. Whitley, A Genetic Algorithm Tutorial, Colorado State University, 1994.

[9] J. M. Baden, and M. N. Cohen, "Optimal Peak Sidelobe Filters for Biphase Pulse Compression”, IEEE International Radar Conference, pp. 249-252, May 1990.

[10] N. Levanon, "Cross-Correlation of Long Binary Signals with Longer Mismatched Filters", IEE Proceedings of Radar Sonar Navigations, Vol. 152, No. 6, pp. 377-382, December 2005.

[11] J. E. Cilliers, and J. C. Smit, "Pulse Compression Sidelobe Reduction by Minimization of Lp-Norms", IEEE Transactions on Aerospace and Electronic Systems, Vol. 43, No. 3, pp. 1238-1247, July 2007.

[12] A. S. Mudukutore, V. Chandrasekar, and R. J. Keeler, "Pulse Compression for Weather Radars", IEEE Transactions on Geosciences and Remote Sensing, Vol. 36, No. 1, pp. 125-142, January 1998. 